\title{
Applicable Prevention Method of Braess Paradox in Urban Traffic Flow Guidance System
}

\author{
Zhaosheng Yang, Peng Gao* \\ State Key Laboratory of Automotive Simulation and Control, School of Traffic, \\ Jilin University, NO.5988 Renmin street, Changchun, 130022, P. R. China \\ Mo Li \\ School of Biological and Agricultural Engnineering, \\ Jilin University, NO.5988 Renmin street, Changchun, 130022, P. R. China \\ Nan Zhang \\ Graduate School of Information Sciences, \\ Tohoku University, Sendai, Miyagi, 980-8579, Japan \\ Received 11 March 2011 \\ Accepted 25 November 2011
}

\begin{abstract}
Urban Traffic Flow Guidance System (UTFGS) may cause the overreaction and concentration of travelers and lead to the phenomenon of Braess Paradox. The paper develops a constrained K-shortest paths algorithm within a dynamic restricted searching area in consideration of realistic road network spatial distribution features. Through the test with the microscopic transportation simulation tool VISSIM, the algorithm could not only decrease the searching scale but also efficiently balance the traffic flow in the net and avoid the phenomenon of Braess Paradox.
\end{abstract}

Keywords: urban traffic flow guidance system, Braess Paradox, K-shortest path optimization, dynamic restricted searching area

\section{Introduction}

Traffic congestion is gradually becoming one of the most serious social problems. One of the most effective solutions to this problem is the establishment of Urban Traffic Flow Guidance System (UTFGS) which could provide the optimum path for travelers before or during the trip based on real-time traffic information in the road network ${ }^{1}$. However, as the overreaction and concentration of drivers, both the static and the dynamic UTFGS may cause a problem of Brass Paradox which may unbalance traffic volume and weaken the effectiveness of navigation information ${ }^{2}$. Even worse, such case will degrade users' trust in the UTFGS and bring very adverse effects to the application and popularization of the navigation technology. For a applicable solution of the Brass Paradox problem in UTFGS, we will first talk about the formation mechanism and prevention method of it at the beginning.

\section{Formation mechanism and applicable prevention method of Braess Paradox}

\subsection{Formation mechanism}

The formation of Braess Paradox problem is closely related to the validity of the traffic guidance information having been released ${ }^{3,4}$. To be specific, the formation mechanism could be concluded as follows:

*Corresponding author: jlugaopeng@ gmail.com 


\subsubsection{Unreasonable traffic assignment proportion}

Without considering the real-time traffic state of all road sections in the network, it is impossible to keep a stochastic user equilibrium assignment of traffic flow and provide appropriate alternative paths to drivers ${ }^{5,6}$.

\subsubsection{Ineffective release strategy of dynamic traffic information}

The release of dynamic traffic information and the improvement of transportation system benefit do not have a positive correlation ${ }^{7,8}$. Ineffective release strategy of dynamic traffic information may cause a disequilibrium of traffic flow that leads to the Braess Paradox. Thus, the determination of appropriate release time, release frequency, and release content of information plays a crucial role in UTFGS ${ }^{9,10}$.

\subsection{Applicable prevention method}

After the analysis, two methods are proposed to prevent the Braess Paradox ${ }^{11,12}$;

\subsubsection{Route choice model in consideration of individual characters}

Analyze the root causes of drives' behavior homoplasy ${ }^{13}$ and establish the route choice model in consideration of individual characters ${ }^{14,15}$, so that paths provided to the drivers could be significantly different between each other and the traffic volume may keep a balance in the road network ${ }^{16,17}$.

\subsubsection{Constrained K-shortest paths algorithm}

While the user group of UTFGS reaches a quasisaturation level, K-shortest path $(K \geq 3)$ with significant differences provided for drivers could not only balance

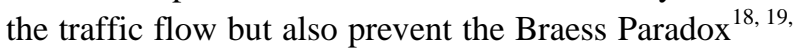
${ }^{20}$. In this paper, we mainly carry some research on this subject.

\section{Constrained K-shortest paths algorithm within a dynamic searching area}

Without considering the constraints in path computation, the existing K-shortest path algorithms may be subdivided in algorithms that allow paths to have repeated links (cycles) ${ }^{21,22,23,24}$ and algorithms that only consider acyclic paths ${ }^{25,26}$. We now consider the problem of finding the K-shortest paths that meet a set of constraints. With a class of constraints, the paper develops Lawlers's ${ }^{25}$ algorithm within a dynamic restricted searching area in consideration of road network spatial distribution features that suitable for travelers.

\subsection{Process of algorithm}

Definition of the dynamic restricted searching area:

(1) Load the data of the road network and initialize the operation environment of the program (Value of $\mathrm{K}$ can be set here with a default value equals to 3);

(2) Set the origin node and destination node of the path computation;

(3) Construct the rectangle with a diagonal line connect the origin and destination to be the restricted searching area of the path computation, set the state variable $I=1$, turn to step (5);

(4) Construct the contour-rectangle of an ellipse with the origin node and destination node as its focus to be the restricted searching area of the path computation, set the state variable $I=2$, turn to step (5);

Initialization of the K-shortest path algorithm:

(5) Define $S_{0,1}$ as the set of all paths from origin to destination with the dynamic restricted searching area;

(6) Find the shortest path within $S_{0,1}$ and denote it with $P\left(S_{0,1}\right)$, set $m=1$;

(7) According to the partitioning rule, divide $S_{0,1}-P\left(S_{0,1}\right)$ into $q(1)$ mutually exclusive subsets. Denote these with $S_{1,1}, S_{1,2}, \ldots, S_{1, q(1)}$, turn to step (9);

Path computation of the K-shortest path algorithm:

(8) Define $S_{a, j}$ as the set that contains the $(m+1) t h$ shortest path. Divide $S_{a, j}-P\left(S_{a, j}\right)$ into $q(m+1)$ mutually exclusive subsets and denote these with $S_{m+1,1}, S_{m+1,2}, \ldots, S_{m+1, q(m+1)}$;

(9) Compute the shortest paths for subsets $S_{m, 1}, S_{m, 2}, \ldots, S_{m, q(m)}$ and denote these paths with $P\left(S_{m, 1}\right), P\left(S_{m, 2}\right), \ldots, P\left(S_{m, q(m)}\right)$;

(10) Find the $(m+1)$ th shortest path within all subsets

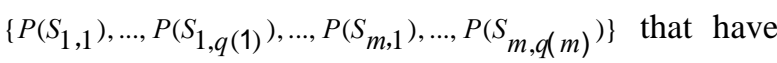
been identfied until now;

(11) Judge whether links contained in the $(m+1) t h$ shortest path satisfy the detour constraint and the overlap constraint. If true, turn to step (9); if not, delete all paths containing these links in set 
$\left\{P\left(S_{1,1}\right), \ldots, P\left(S_{1, q(1)}\right), \ldots, P\left(S_{m, 1}\right), \ldots, P\left(S_{m, q(m)}\right)\right\}$, then turn to step (12);

(12) Judge whether the set $\left\{P\left(S_{1,1}\right), \ldots, P\left(S_{1, q(1)}\right), \ldots, P\left(S_{m, 1}\right), \ldots, P\left(S_{m, q(m)}\right)\right\}$ equals to null. If true, turn to step (13); if not, turn to step (10);

(13) Jude whether the state variable $I=1$. If true, turn to step(4); if not, turn to step(16);

(14) Bring this path to the set of K-shortest paths, set $m=m+1$;

(15) Jude whether $m=K$. If true, turns to step (16); if not, turn to step (8);

(16) Terminate this algorithm and output all paths containing in the set of K-shortest paths to drivers.

The complexity of the algorithm for finding Kshortest paths is $O\left(k^{*} n^{*} c(s p t)\right)$, with $n$ the number of nodes within the restricted searching area and $c(s p t)$ the complexity of the single source shortest path algorithm.

\subsection{Restricted searching area of the path computation}

The constrained K-shortest paths algorithm uses the dijkstra algorithm as a core to compute the single source shortest path. As the dijkstra algorithm is a traversal one, the CPU time of the path computation may not be satisfied with the real-time demand of UTFGS when the scale of road network becomes very large.

Based on the statistical analysis of the spatial distribution of real road network, two restricted searching areas with the appropriate restricted rectangle and contour rectangle of an ellipse are established. With this restriction, the algorithm can efficiently decrease the searching scale and improve its running efficiency.

(1) Rectangle with a diagonal line connects the origin and destination.

Restrict the searching area in the rectangle with a diagonal line connects the origin and destination, and then compute the K-shortest path in this area. If quantity of computed alternatives paths could not reaches the presupposition value $\mathrm{K}$, turn to the restricted area of the contour-rectangle of an ellipse and continue.

(2) Contour-rectangle of an ellipse with the origin and destination as its focus.

Total length from the point on an ellipse to its focus equals a fixed value. With the statistical analysis of the road network spatial distribution features, an ellipse with the origin and destination as its focus could be constructed as follows ${ }^{27,28}$ :

$$
\begin{gathered}
A=P_{O D} /\left(2 L_{O D} \sqrt{\left(y_{D}-y_{S}\right)^{2}+\left(x_{D}-x_{S}\right)^{2}}\right), \\
B=\sqrt{A^{2}-\left(\left(y_{D}-y_{S}\right)^{2}+\left(x_{D}-x_{S}\right)^{2}\right) / 2},
\end{gathered}
$$

Where, $\left(x_{s}, y_{S}\right)$ and $\left(x_{D}, y_{D}\right)$ respectively stand for the coordinates of the origin node and destination node; $A$ and $B$ respectively stand for length of the long axis and short axis of ellipse; $L_{O D}$ and $P_{O D}$ respectively stand for the Euclidean distance and the shortest path length between the origin node and the destination node; $\tau=R_{95 \%}=\left(P_{O D} / L_{O D}\right)_{95 \%}$ stands for the value of $R_{O D}$ under a confidence level of $95 \%$.

In order to avoid large amount of power and evolution operation in computation, the above area could be extended to the contour-rectangle of the former ellipse:

$$
\begin{aligned}
& x=\left(x_{O}+x_{D}\right) / 2 \pm \sqrt{A^{2} \cos ^{2} \theta+B^{2} \sin ^{2} \theta}, \\
& y=\left(y_{O}+y_{D}\right) / 2 \pm \sqrt{A^{2} \sin ^{2} \theta+B^{2} \cos ^{2} \theta}
\end{aligned}
$$

Where, the extreme value of $x, y$ stand for the contour-rectangle of the ellipse with the origin node and destination node as its focus.

\subsection{Partitioning rule}

Let $\mathrm{S}$ be the set of acyclic paths between origin and destination that all start with a sequence of links denoted as $L^{\text {initial }}=\left\{b_{1}, b_{2}, \ldots, b_{G}\right\}$ and exclude the links contained in $L^{\text {exclude }}=\left\{c_{1}, c_{2}, \ldots ., c_{H}\right\}$, and define $P(S)$ as the shortest path consists of the links $\left\{\left\{L^{\text {initial }}\right\}, a_{1}, a_{2}, \ldots ., a_{q}\right\}$. Then the algorithm divides the set $S-P(S)$ into $q$ mutually subsets $S_{1}, S_{2}, \ldots, S_{q}$, where $S_{i}$ is the set of acyclic paths between origin and destination that start with the sequence of links $L_{i}^{\text {initial }}=\left\{\left\{L^{\text {initial }}\right\}, a_{1}, a_{2}, \ldots, a_{i-1}\right\} \quad$ and exclude the links $L_{i}^{\text {exclude }}=\left\{\left\{L^{\text {exclude }}\right\}, a_{i}\right\}$.

So, computing the shortest path in $S_{i}$ could be a straightforward task: simply compute the shortest path from the end node of $a_{i-1}$ to the destination, while excluding the links contained in $L_{i}^{\text {exclude }}=\left\{\left\{L^{\text {exclude }}\right\}, a_{i}\right\}$ and the predecessor links of $a_{i-1}$ given by $L_{i}^{\text {initial }}=\left\{\left\{L^{\text {initial }}\right\}, a_{1}, a_{2}, \ldots ., a_{i-2}\right\}$. 


\subsection{Constraints}

To satisfy the actual demands of drivers, two constraints are applied to eliminate the unreasonable paths. In each computation, only the paths satisfy the constraints could be brought to the K-shortest paths set and finally provided for drives.

Before the intruoducation of containts, some conceptions must be defined.

Denote each path with a node series $(i, j, k, m, \ldots$.$) .$ Define set of the mutually exclusive links consist in a certain path as incomplete link set and denote this set with $S_{A}=\{i-j, j-k, k-m, \ldots\}$. Define set of the full permutation mutually exclusive links consist in a certain path as complete link set and denote this set with $S_{B}=\{i-j, i-k, i-m, \ldots, j-k, j-m, \ldots, k-m, \ldots\}$. Define length of links start from node $i$ and terminate at node $j$ through a series of nodes as $l(i-j)$, where $i-j$ represents the meaning of road ID.

\section{(1)Detour constraint}

All paths that contain a detour larger than a fator $\phi^{\max }$ relative to the shortest path must be eliminateed.

In the computation of the $(m) t h$ shortest path, compare the complete link set $S_{B(i, q(j))}$ originated from $P\left(S_{i, q(j)}\right)$ with complete link set $S_{B(0,1)}$ originated from $P\left(S_{0,1}\right)$. If there exist two links sharing the same ID, the further step compare the length of each link it represents in its set.

If path $P\left(S_{i, q(j)}\right)$ satisfies the formula $l_{i, j}(s-t)>\phi^{\max } L(s-t)$, it is not reasonable from a behavioral point of view, all paths contain link $s-t$ will be eliminateed later.

Where, $\phi^{\max }$ stands for detour constraint factor; $l_{i, j}(s-t)$ stands for length of the link in $S_{B(i, q(j))}$ that shares the same ID; $L(s-t)$ stands for length of the link in $S_{B(0,1)}$ that shares the same ID.

\section{(2)Overlap constraint}

For a significant difference between each alternative path, all paths that contain a overlap larger than a fator $\varphi^{\min }$ relative to the shortest path must be eliminateed.

In the computation of the $(m) t h$ shortest path, compare the incomplete link set $S_{A(i, q(j))}$ originated from $P\left(S_{i, q(j)}\right)$ with all the incomplete link set $S_{A(0,1)}, S_{A(1,1)}, \ldots, S_{A(m-1,1)}$ originated from the former K-shortest path $(K=1, \ldots, m-1)$. If there exist two links sharing the same ID, the further step sum the length of relative link it represents in set $S_{A(i, q(j))}$.

If path $P\left(S_{i, q(j)}\right)$ satisfies the formula $\sum l_{i, j}^{r}(s-t)>\varphi{ }^{\min } L_{K=1}$, it is not reasonable from a behavioral point of view, all paths contain link $s$ - $t$ will be eliminateed later.

Where, $\varphi^{\min }$ stands for overlap constraint factor; $l_{i, j}^{r}(s-t)$ stands for length of the link in $S_{A(i, q(j))}$ compared with $S_{A(r, 1)}$ that shares the same ID; $L_{K=1}$ stands for length of the overall shortest path $P\left(S_{0,1}\right)(K=1)$.

\section{Realization of algorithm}

Realize the algorithm in environment of Changchun. The sample distribution ratio coefficient under a confidence level of $95 \%$ could be statistics obtained in Fig.1. Set the detour constraint $\phi^{\max }=1.25$, overlap constraint $\varphi^{\min }=0.5$ in the initialization interface of Fig.2 and run the program on HP xw4600 workstation with the CPU frequency of $2.2 \mathrm{GHz}$ and the physical memory of 2G. The operation effect of the K-shortest paths $(K=3)$ start from node 2618 and terminate at node 97 are shown in Fig.3. The CPU running time is $0.58 \mathrm{~s}$ in contrast with $7.32 \mathrm{~s}$ of constraint K-shortest path algorithm without restricted searching area.

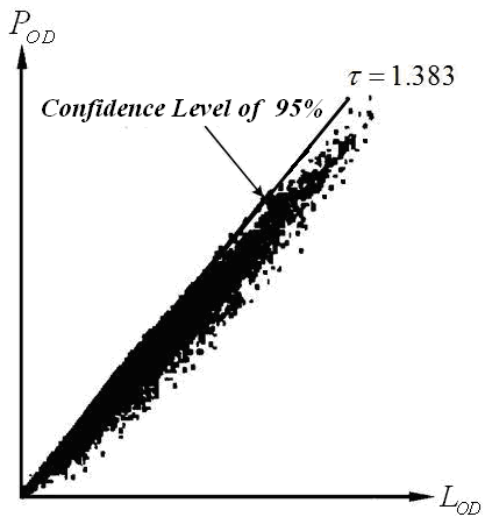

Fig. 1. Sample Distribution Ratio Coefficient of Changchun. 


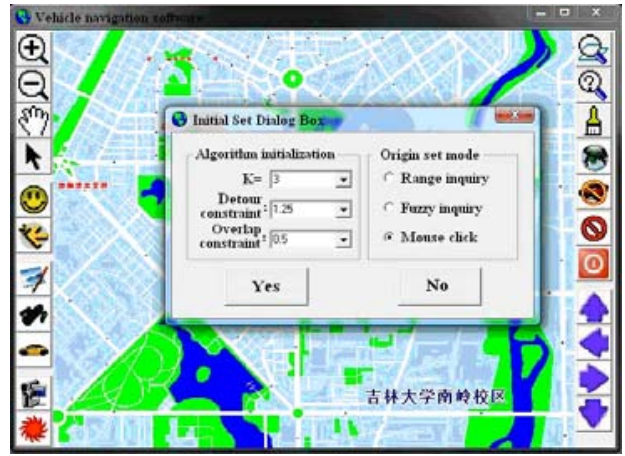

Fig. 2. Initialization interface of the algorithm.

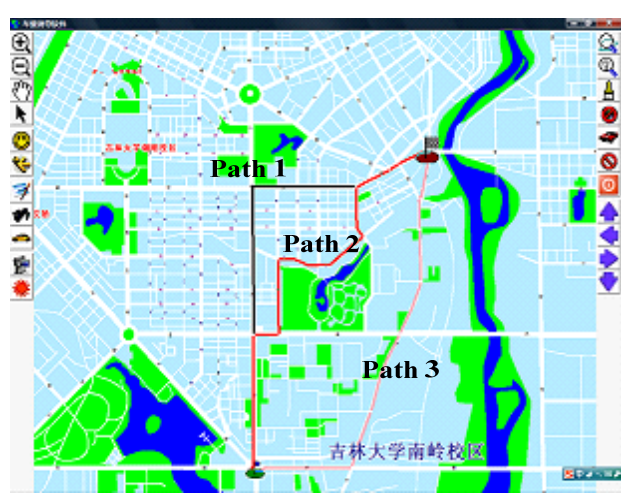

Fig. 3. Operation effect of the algorithm.

Meanwhile, comparisons of searching area with and without restrictions are shown in Fig. 4 where the red nodes represent the searching set during path calculation.

The proposed algorithm has not only effectively decreased the searching scale but also dramatically increase the path optimization efficiency for about $92 \%$.

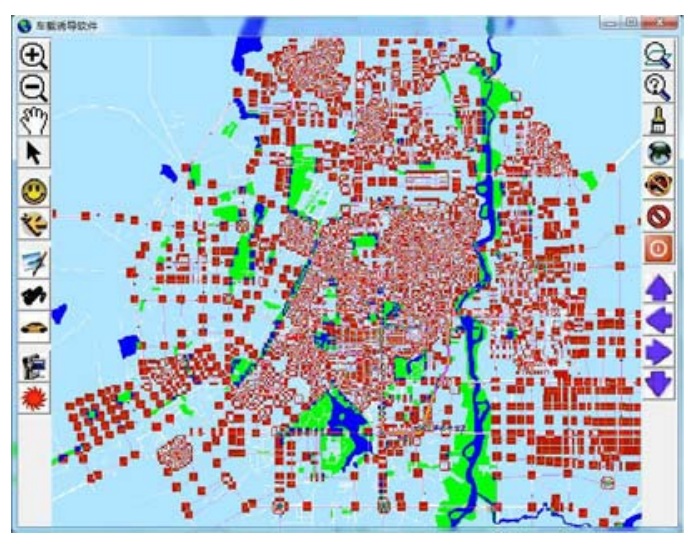

(a) Searching area without restrictions

\section{Test of algorithm}

\subsection{Parameter sensitivity test}

According to different sizes of road network $(N=100,200,400)$ and solution space $(K=5,10,15,20)$, analyze the parameter sensitivity of detour constraint $\phi^{\max }$ and overlap constraint $\varphi^{\min }$ by taking the different proportion value $(80 \%, 100 \%, 120 \%)$ of $\phi_{100 \%}^{\max }=1.25, \quad \varphi_{100 \%}^{\min }=0.5$ which is shown in Table 1.

Table 1. Parameter sensitivity test of constraint factors .

\begin{tabular}{c|c|c|c|c|c|c|}
\hline $\mathrm{N}$ & $\mathrm{K}$ & $\begin{array}{c}\varphi_{100 \%}^{\min } \\
\phi_{\max }\end{array}$ & $\begin{array}{c}\varphi_{80 \%}^{\min } \\
\phi_{\max }\end{array}$ & $\begin{array}{c}\varphi_{120 \%}^{\min } \\
\phi_{\max }\end{array}$ & $\begin{array}{c}\varphi_{100 \%}^{\min } \\
\phi_{\max }\end{array}$ & $\begin{array}{c}\varphi_{100 \%} \\
{ }_{\max } \\
\phi_{120 \%}\end{array}$ \\
\hline 100 & 50 & 4 & $3(78 \%)$ & $4(113 \%)$ & $3(81 \%)$ & $4(97 \%)$ \\
\hline$\ldots$ & 100 & 7 & $6(78 \%)$ & $8(116 \%)$ & $6(83 \%)$ & $7(103 \%)$ \\
\hline$\ldots$ & 150 & 11 & $8(75 \%)$ & $13(123 \%)$ & $9(82 \%)$ & $11(105 \%)$ \\
\hline$\ldots$ & 200 & 14 & $11(75 \%)$ & $19(130 \%)$ & $12(84 \%)$ & $16(111 \%)$ \\
\hline$\ldots$ & 50 & 11 & $9(82 \%)$ & $17(146 \%)$ & $11(96 \%)$ & $13(114 \%)$ \\
\hline$\ldots$ & 100 & 25 & $20(19 \%)$ & $35(138 \%)$ & $24(93 \%)$ & $26(104 \%)$ \\
\hline$\ldots$ & 150 & 41 & $32(78 \%)$ & $57(137 \%)$ & $41(99 \%)$ & $42(101 \%)$ \\
\hline$\ldots$ & 200 & 59 & $44(74 \%)$ & $77(131 \%)$ & $58(97 \%)$ & $60(101 \%)$ \\
\hline$\ldots$ & 150 & 27 & $3(11 \%)$ & $30(114 \%)$ & $25(92 \%)$ & $26(98 \%)$ \\
\hline$\ldots$ & 200 & 115 & $96(84 \%)$ & $141(122 \%)$ & $112(97 \%)$ & $116(101 \%)$ \\
\hline
\end{tabular}

Two aspects of conclusions have been drawn:

(1) Keep the value of $\varphi^{\min }$ constant. The larger $\phi^{\max }$ is, the more paths that do not meet detour constraint appear and the more time it takes for CPU runs. (2)Keep the value of $\phi^{\max }$ constant. The larger $\varphi^{\min }$ is, the more paths that do not meet detour constraint appear and the more time for CPU runs.

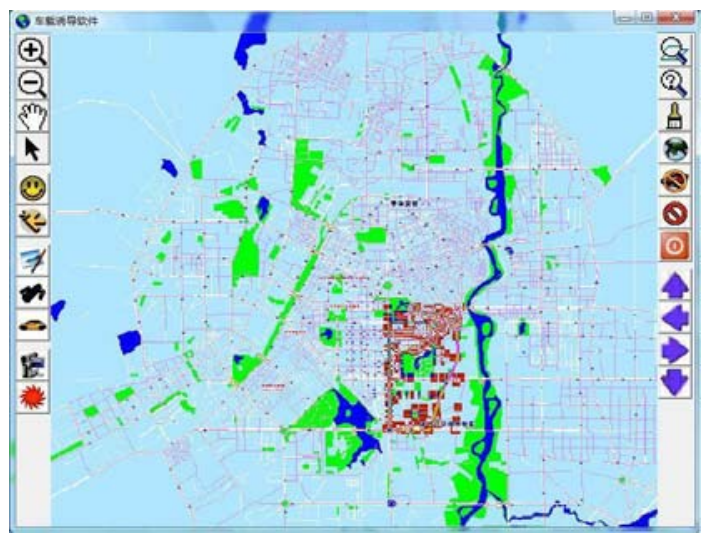

(b) Searching area with restrictions

Fig. 4. Comparison of searching area 


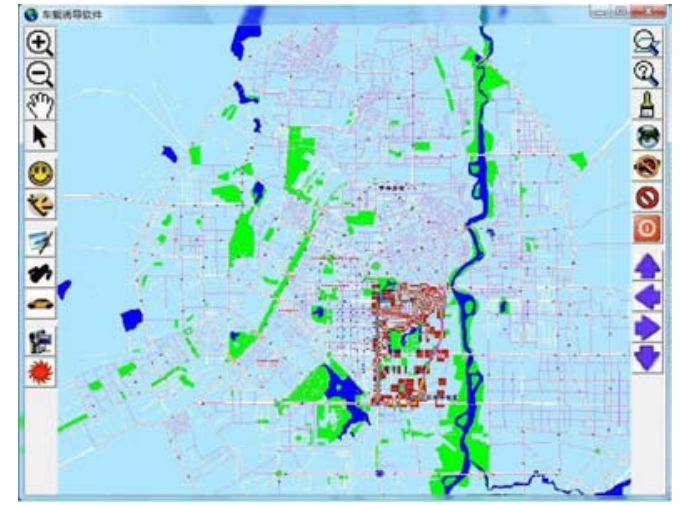

(a) Searching area of $\phi^{\max }=1.25, \varphi^{\min }=0.5$

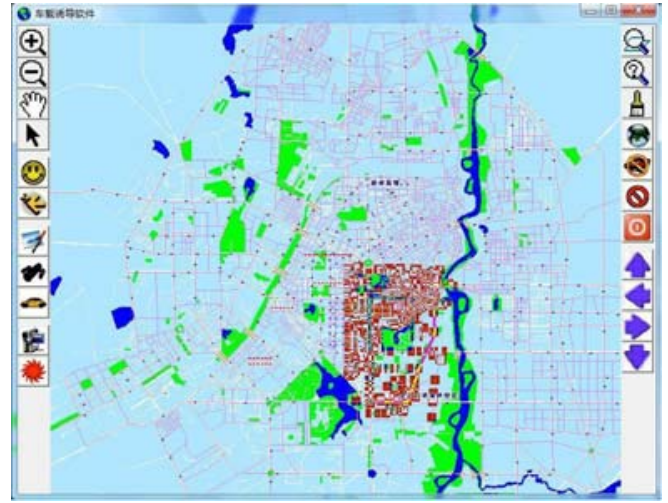

(b) Searching area of $\phi^{\max }=1.25, \varphi^{\min }=0.4$

Fig. 5. Dynamic switch of searching area.

On setting $\phi^{\max }=1.25, \varphi^{\min }=0.4$, the overlap become tight constraint. Diagonal rectangle in Fig.3 could no longer meet the searching need of $K=3$, the state variable was assigned equal to 2 and the search area is therefore switched to the contour-rectangle of the ellipse (right part) shown in Fig.5

\subsection{Operating efficiency test in large network}

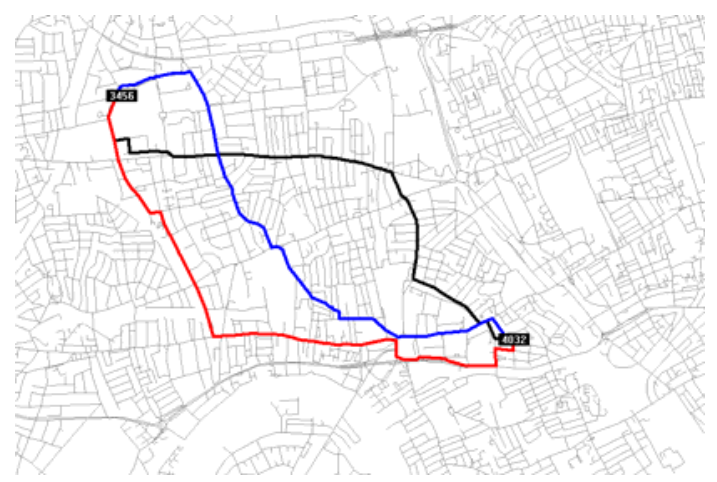

Fig. 6. Efficiency test in Guangzhou network.

Test the algorithm efficiency in large-scale road network $^{29}$ of Guangzhou which is shown in Fig.6 and
Table 2. From the comparison of results, operating efficiency of the algorithm is improved by about $89.6 \%$ under a reasonable restricted searching area.

\subsection{Network benefit test}

Construct the simulating road network shown in Fig.7 with VISSIM 5.1. Set the simulation scheme ${ }^{30}$ under the restriction of detour constraint $\phi^{\max }=1.25$, overlap constraint $\varphi^{\min }=0.5$. Set the simulation time to 3600s and the sampling interval to 100s. The simulated traffic incident starts at 600s and lasts to the end. Collect all the parameters including travel time, queue length, average vehicle delay, etc that related to the effect evaluation of traffic guidance. Evaluate the road network benefit with the total travel cost. The total travel cost of the simulated road network without any prevention method being carried out is 35903950s (Braess Paradox appears) in contrast with 24397420s that using the algorithm. During the whole simulation process that using the algorithm, the former congestion of dissipated and no significant Braess Paradox phenomenon appears.

Table 2. Efficiency test in Guangzhou network.

\begin{tabular}{ccccccc}
\hline & $\begin{array}{c}\text { Origin } \\
\text { Node }\end{array}$ & $\begin{array}{c}\text { Destination } \\
\text { Node }\end{array}$ & $\begin{array}{c}\text { CPU running } \\
\text { time without } \\
\text { restricted } \\
\text { searching area }\end{array}$ & $\begin{array}{c}\text { CPU running } \\
\text { time without } \\
\text { restricted } \\
\text { searching area }\end{array}$ & $\begin{array}{c}\text { Network } \\
\text { searching } \\
\text { area without } \\
\text { restriction }\end{array}$ & $\begin{array}{c}\text { Network } \\
\text { searching } \\
\text { area with } \\
\text { restriction }\end{array}$ \\
\hline 1 & 171 & 10839 & 15.015 & 1.062 & 21658 & 4576 \\
2 & 356 & 658 & 17.910 & 1.921 & 24338 & 4064 \\
3 & 555 & 10839 & 17.343 & 2.031 & 26160 & 8021 \\
4 & 666 & 10912 & 2.225 & 0.370 & 2562 & 623 \\
5 & 8520 & 777 & 5.980 & 0.901 & 6737 & 2215 \\
6 & 332 & 8520 & 15.895 & 1.140 & 21139 & 2481 \\
7 & 298 & 12 & 12.110 & 0.686 & 16554 & 1515 \\
8 & 298 & 999 & 15.640 & 1.484 & 24116 & 3185 \\
\hline
\end{tabular}




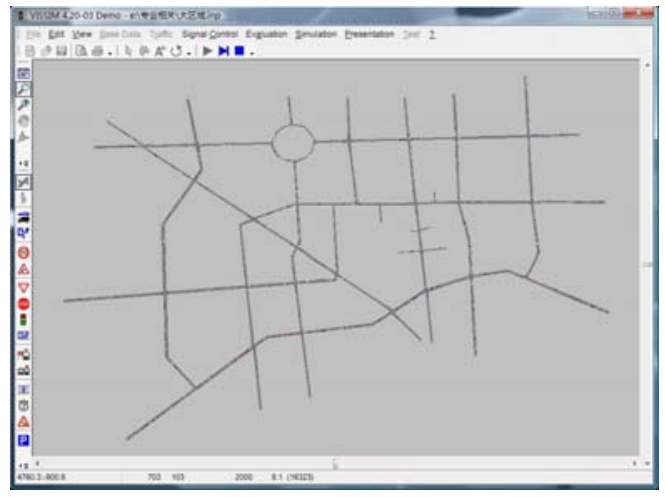

Fig. 7. Simulation network of VISSIM.

\section{Conclusions}

With the constraints of detour links and overlap links, this paper develops a constrained K-shortest paths algorithm within a dynamic restricted of searching area in consideration of road network spatial distribution features which is suitable for travelers. The algorithm can not only decrease the searching scale but also efficiently balance the traffic flow and applicably prevent the Braess Paradox problem. However, as the little consideration of individual route choice behavior, this subject still needs some further study in future.

\section{Acknowledgements}

The author expresses his sincere appreciation to the Chinese National High Technology Research and Development Program Committee for the financial support of this subject under grants No. 2009AA11Z208 and 2009AA11Z218.

\section{References}

1. C. Little, The Intelligent Vehicle Initiative: Advancing "Human-Centered" Smart Vehicles, Public Roads, 61(2) (1997)18-25

2. Z.S. Yang, Urban Traffic Flow Guidance System, (China railway publishing house, Beijing, 2004).

3. R. Arnott, Does Providing Information to Driver Reduce Traffic Congestion?, Transportation Research. 25A(5) (1991)309-318.

4. Ben-Akiva, André de Palma and Isam Kaysi, Dynamic Network Models and Driver Information Systems, Transportation Research. 25A(5) (1991)251-266.

5. L. R. Rilett, Van Aerde, Modeling distributed real-time route guidance strategies in a traffic network that exhibits the Braess paradox, Vehicle Navigation \& Information Systems Conference Proceedings, IEEE, SAE Dearborn, Michigan. (1991)577-587.
6. A. Hallefjord, K. Jornsten, S. Storoy, Traffic equilibrium paradoxes when travel demand is elastic. Asia-Pacific Journal of Operational Research, (1994) 41-50.

7. E. I. Pas, S. L. Principio, Braess' paradox: some new insights. Transportation Research 31B (1997)265-276.

8. C. Daganzo, Two paradoxes of traffic flow on networks with physical queues. II Symposium Ingenieria de los Transportes. (1996) 55-62.

9. G. Y. Jiang, Z.D. Zheng, Z. Bai, Causes and preventive technologies of traffic congestion shift, Journal of Jilin University: Engineering and Technology Edition. 7(4) (2007) 93-97.

10. W. H. Randolph, Route Choice and Advanced Traveler Information Systems on a Capacitated and Dynamic Network, Transportation Research. 4C(5) (1996) 289306.

11. W. Barfield and T. A. Dingus, Human factors in intelligent transportation systems(Lawrence Erlbaum Associates, Inc. New Jersey, 1997)

12. W. H. Wang, Driving Behaviour Theory and Method in Road Transport System (Science Press, Beijing, 2001)

13. M. Z. Jin, X. Lei, J. Du, Evolutionary Game Theory in Multi-Objective Optimization Problem, International Journal of Computational Intelligence Systems, 3(suppl1)(2010),74-87.

14. M. G. Flyte, The safety design of in-vehicle information and support system: the human factors issues, International journal of vehicle design, 16(1995)158-169.

15. C. Daganzo, Queue spillovers in transportation networks with a route choice. Transportation Science. 32(1998) 311.

16. Yasunori Iida, Takamasa Akiyama and Takashi Uchida, Experimental Analysis of Dynamic Route Choice Behavior, Transportation Research. 26B (1992)17-32.

17. L. Takayama and C. Nass, Driver safety and information from afar: An experimental driving simulator study of wireless vs. in-car information services, Int. J. HumanComputer Studies 66 (2008) 173-184.

18. T. A. Ranney, Models of driving behaviour: A review of their evolution, Accident analysis and prevent, 26(1994), 733-750.

19. W. H. Wang, F. U. Huo, H. C Tan, H. Bubb, A Framework for Function Allocation in Intelligent Driver Interface Design for Comfort and Safety, International Journal of Computational Intelligence Systems, 3(5)(2010)531-541.

20. J. Rasmussen, Skills, rules, knowledge; signals, signs, and symbols, and other distinctions in human performance models. IEEE Transactions on Systems, Man and Cybernetics, 13(1983)257-266.

21. R. Bellman, R. Kabala, On kth best policies, Journal of SIAM. 8(1960)582-585.

22. D. Eppstein, Finding the K shortest paths, Foundations of Computer Science. (1994)54-165.

23. W. Hoffman, R. Pavley, A method for the solution of the nth best path problem, Journal of the Association for Computing Machinery. 6(1959)506-514. 
24. R. D. Shier, On algorithms for finding the $\mathrm{K}$ shortest paths in a network, Networks. 7(1979)341-352.

25. E. L. Lawler, Combinatorial Optimization: Networks and Matroids (Courier Dover Publications, New York, 1976)

26. J. Y. Yen, Finding the $\mathrm{K}$ shortest loopless paths in a network, Management Science. 17 (1971)716-721.

27. F. Lu, S.M. Lu, Time Shortest Path Algorithm for Restricted Searching Area in Transportation Networks, Journal of Image and Graphics, 4(10) (1999)850-853.

28. F. Lu, C. H. Zhou, Q Wan. An Optimum Path Algorithm for Traffic Network Based on Hierarchical Spatial Reasoning, Geospatial Information Science. 3(4)(2000)36-42.

29. E. B. David, D. H. Lee, N. J. Bruce, Dynamic Route choice model of large-scale traffic network, Journal Of Transportation Engineering. 123(4)(1997) 276-282.

30. R. Sukthankar, J. Hancock, C. Thorpe, Tactical-level Simulation for Intelligent Transportation Systems, Mathematical and Computer Modelling, 27(1998), 19-24. 